\title{
Quasi-local conserved charges in Lorenz-diffeomorphism covariant theory of gravity
}

\author{
H. Adami ${ }^{\mathrm{a}}$, M. R. Setare ${ }^{\mathrm{b}}$ \\ Department of Science, University of Kurdistan, Sanandaj, Iran
}

Received: 4 February 2016 / Accepted: 20 March 2016 / Published online: 6 April 2016

(C) The Author(s) 2016. This article is published with open access at Springerlink.com

\begin{abstract}
In this paper, using the combined Lorenzdiffeomorphism symmetry, we find a general formula for the quasi-local conserved charge of the covariant gravity theories in a first order formalism of gravity. We simplify the general formula for the Lovelock theory of gravity. Afterwards, we apply the obtained formula on BHT gravity to obtain the energy and angular momentum of the rotating OTT black hole solution in the context of this theory.
\end{abstract}

\section{Introduction}

The concept of conserved charges is a very important matter in gravity theories as well as in other physical theories. As is well known, the concept of conserved charges of gravity theories is related to the concept of the Noether charges corresponding to the Killing vectors which are admitted by solutions of a theory. There are several approaches to obtain the mass and angular momentum of black holes solutions of different gravity theories [1-18]. According to the Arnowitt, Deser, and Misner formalism (ADM formalism) [3] one can obtain the conserved charges of an asymptotically flat spacetime solution of a general theory of relativity, but this is not a covariant method. The ADM formalism has been extended to include asymptotically AdS spacetime solution of Einstein gravity [1,2]. Deser and Tekin have extended this approach. By this extension one can calculate the energy of asymptotically dS or AdS solutions in higher curvature gravity models and also in a topologically massive gravity model [4-6]. This method is a covariant formalism; it is known as the Abbott-Deser-Tekin (ADT) formalism [1,2,4-6]. Thus the ADT formalism is applicable in the general higher curvature and higher derivative theories of gravity [4-19]. Another method is the Brown-York formalism [7], which is based on a quasi-local concept, but this approach also is not covari-

\footnotetext{
a e-mail: hamed.adami@yahoo.com

be-mail: rezakord@ipm.ir
}

ant. A general definition of conserved charges in general relativity and other theories of gravity has been proposed in [20,21], but this approach is applicable for asymptotically flat spacetime solutions. This formalism has been extended to non-covariant theories by Tachikawa [22].

Wontae Kim et al. [8] have proposed a way to calculate the conserved charges of all non-asymptotically flat solutions of covariant theories of gravity as well as asymptotically flat ones, which is based on the concept of the quasi-local conserved charges. This formalism is established on a metric formalism of gravity theories and has been extended to noncovariant theories in [9]. But it is well known that we can write down the gravity theories in the first order formalism. In the first order formalism of gravity theories, there are some theories which are not only diffeomorphism covariant but also covariant under the local Lorenz gauge transformations. The authors in Ref. [23] have combined these two symmetries in an appropriate way and they obtained a new combined symmetry, the "Lorenz-diffeomorphism symmetry". In that paper, the authors have shown that the entropy of black holes in the covariant theories of gravity is simply a Lorenzdiffeomorphism Noether charge. Recently, this method was extended to include non-covariant theories of gravity [26].

Our approach has some similarity with what one can do in the framework of Poincaré gauge theory [27-32] (see also $[33,34]$ for recent work on this topic). Poincaré gauge symmetry is the symmetry of Poincare gauge theory. In the framework of this theory the translation and Lorentz symmetry are local. Here we combine the Lorenz gauge transformation with a diffeomorphism, then we introduce the total variation under a Lorenz-diffeomorphism transformation motivated by work of Jacobson and Mohd [23]. In this case we have a unique transformation, and using it we can obtain conserved charges of a covariant theory of gravity in the first order formalism.

Our aim in this paper is to find quasi-local conserved charges of covariant theories of gravity in the first order for- 
malism. For this purpose, we use the Lorenz-diffeomorphism symmetry. In this method the quasi-local conserved charges are Lorenz-diffeomorphism Noether charges associated to the Killing vectors which are admitted by the considered spacetime. The advantages of this proposal are, first, that the quasi-local conserved charges are calculated off-shell; second, we can calculate these for spacetime solutions which are not asymptotically flat (or even AdS).

Our paper is organized as follows. In Sect. 2 using the Lorentz-Lie (L-L) derivative, we obtain the total variation of the vielbein and the spin connection. In Sect. 3 we consider the most general Lorenz-diffeomorphism invariant Lagrangian $n$-form of a gravity theory and obtain a general formula for quasi-local conserved charges. In Sect. 4 we apply our method on the Lovelock theory in arbitrary dimensions and find a general expression for the conserved charges of this theory in any dimension. In Sect. 5, using the provided formalism, we find the energy and angular momentum of the rotating OTT black hole solution of BHT gravity. Also, we calculate the entropy of this black hole using the general formula for the Chern-Simons-like theories of gravity [26]. Section 6 is devoted to conclusions and discussions.

\section{Lorentz-Lie derivative and total variation}

Consider a $n$-dimensional spacetime. Let $e_{\mu}^{a}$ denote the vielbein, thus we can write the metric as $g_{\mu \nu}=\eta_{a b} e_{\mu}^{a} e_{\nu}^{b}$ where $\eta_{a b}$ denotes the Minkowski metric. Under a Lorenz gauge transformation $\Lambda \in S O(n-1,1)$ the vielbein transforms as $\tilde{e}_{\mu}^{a}=\Lambda_{b}^{a} e_{\mu}^{b}$ so that the spacetime metric under this transformation remains unchanged. In the first order formalism, the spin connection is treated as an independent quantity, like the vielbein, and it is denoted by $\omega_{\mu}^{a b}$. Under a Lorenz gauge transformation the spin connection transforms as $\tilde{\omega}=\Lambda \omega \Lambda^{-1}+\Lambda \mathrm{d} \Lambda^{-1}$, so this is not an invariant quantity under the considered transformation but we know that under general coordinate transformations the spin connection transforms as a covariant vector. One can define the vielbein 1-form and the spin-connection 1-form as $e^{a}=e_{\mu}^{a} \mathrm{~d} x^{\mu}$ and $\omega^{a b}=\omega_{\mu}^{a b} \mathrm{~d} x^{\mu}$, respectively. The Lorentz-Lie (L-L) derivative of the vielbein 1 -form is defined as [23] $]^{1}$

$\mathfrak{L}_{\xi} e^{a}=\mathfrak{f}_{\xi} e^{a}+\lambda_{b}^{a} e^{b}$,

where $£_{\xi}$ denotes the ordinary Lie derivative along $\xi$ and $\lambda_{b}^{a}$ generates the Lorenz gauge transformations $S O(n-1,1)$. In general, $\lambda_{b}^{a}$ is independent from the vielbein and spin connection and it is a function of spacetime coordinates and of the diffeomorphism generator $\xi$. It is straightforward to extend

1 This is known in the literature as the "generalized Lie derivative" $[24,25]$. this expression of the $\mathrm{L}-\mathrm{L}$ derivative for $e^{a}$ to the case for which we have more than one Lorentz index. Now, the total variation under a diffeomorphism, which is generated by a vector field $\xi$, is considered as a combination of a variation with respect to the infinitesimal coordinates and the infinitesimal Lorenz gauge transformation, which is constructed out of the diffeomorphism generator $\xi$. Hence, the total variation of the vielbein and the spin connection are [26]

$\delta_{\xi} e^{a}=\mathfrak{L}_{\xi} e^{a}$,

$\delta_{\xi} \omega^{a b}=\mathfrak{L}_{\xi} \omega^{a b}-\mathrm{d} \lambda^{a b}$,

respectively. The extra term in (3), $-\mathrm{d} \lambda^{a b}$, may be the origin of a non-covariant theory, but here this does not stand in our way because we only focus on covariant theories.

\section{A general formula for the quasi-local conserved charges}

In this section, we try to find a general formula for quasi-local conserved charges of a covariant theory of gravity in the first order formalism. As is well known, the curvature 2-form and the torsion 2-form are defined, respectively, as

$R^{a b}=\mathrm{d} \omega^{a b}+\omega_{c}^{a} \wedge \omega^{c b}$,

$T^{a}=D e^{a}=\mathrm{d} e^{a}+\omega_{b}^{a} \wedge e^{b}$,

where $D$ denotes the exterior covariant derivative. We consider the following $n$-form Lorenz-diffeomorphism invariant Lagrangian of a gravity theory:

$L=L(e, T, R, f, h)$,

where $f^{a}$ and $h^{a}$ are Lorenz-diffeomorphism covariant 1form fields. By varying the above Lagrangian with respect to the fields, we will have

$$
\begin{aligned}
\delta L= & \delta e^{a} \wedge E_{a}^{(e)}+\delta \omega^{a b} \wedge E_{a b}^{(\omega)}+\delta f^{a} \wedge E_{a}^{(f)} \\
& +\delta h^{a} \wedge E_{a}^{(h)}+\mathrm{d} \Theta(\Phi, \delta \Phi) .
\end{aligned}
$$

The equations of motion are $E_{a}^{(e)}=E_{a b}^{(\omega)}=E_{a}^{(f)}=$ $E_{a}^{(h)}=0$ and $\Theta(\Phi, \delta \Phi)$ is simply the surface term, where $\Phi=\{e, \omega, f, h\}$. If this variation is due to a diffeomorphism which is generated by the vector field $\xi$ then the total variation of Lagrangian (6) with respect to the diffeomorphism $\xi$ is

$$
\begin{aligned}
\delta_{\xi} L= & \delta_{\xi} e^{a} \wedge E_{a}^{(e)}+\delta_{\xi} \omega^{a b} \wedge E_{a b}^{(\omega)}+\delta_{\xi} f^{a} \wedge E_{a}^{(f)} \\
& +\delta_{\xi} h^{a} \wedge E_{a}^{(h)}+\mathrm{d} \Theta\left(\Phi, \delta_{\xi} \Phi\right) .
\end{aligned}
$$

On the other hand, using (4), one can rewrite (2) and (3) as follows:

$\delta_{\xi} e^{a}=D i_{\xi} e^{a}+i_{\xi} T^{a}+\left(\lambda^{a b}-i_{\xi} \omega^{a b}\right) e_{b}$,

$\delta_{\xi} \omega^{a b}=i_{\xi} R^{a b}+D\left(i_{\xi} \omega^{a b}-\lambda^{a b}\right)$, 
respectively. Also we have

$$
\begin{aligned}
& \delta_{\xi} f^{a}=D i_{\xi} f^{a}+i_{\xi} D f^{a}+\left(\lambda^{a b}-i_{\xi} \omega^{a b}\right) f_{b}, \\
& \delta_{\xi} h^{a}=D i_{\xi} h^{a}+i_{\xi} D h^{a}+\left(\lambda^{a b}-i_{\xi} \omega^{a b}\right) h_{b},
\end{aligned}
$$

where $i_{\xi}$ denotes the interior product in $\xi$. By substituting Eqs. (8)-(10) into Eq. (7), we have

$$
\begin{aligned}
\delta_{\xi} L & =\mathrm{d}\left(i_{\xi} e^{a} E_{a}^{(e)}+i_{\xi} f^{a} E_{a}^{(f)}+i_{\xi} h^{a} E_{a}^{(h)}\right. \\
& \left.+\left(i_{\xi} \omega^{a b}-\lambda^{a b}\right) E_{a b}^{(\omega)}+\Theta\left(\Phi, \delta_{\xi} \Phi\right)\right)+\left(\lambda^{a b}-i_{\xi} \omega^{a b}\right) \\
& \times\left(D E_{a b}^{(\omega)}+e_{b} \wedge E_{a}^{(e)}+f_{b} \wedge E_{a}^{(f)}+h_{b} \wedge E_{a}^{(h)}\right) \\
& -i_{\xi} e^{a} D E_{a}^{(e)}-i_{\xi} f^{a} D E_{a}^{(f)}-i_{\xi} h^{a} D E_{a}^{(h)} \\
& +i_{\xi} D f^{a} \wedge E_{a}^{(f)}+i_{\xi} D h^{a} \wedge E_{a}^{(h)} \\
& +i_{\xi} T^{a} \wedge E_{a}^{(e)}+i_{\xi} R^{a b} \wedge E_{a b}^{(\omega)} .
\end{aligned}
$$

As we expect, the last two lines in the above expression can be rewritten in the following form:

$$
i_{\xi} e^{a} X_{a}(e, \omega, f)+i_{\xi} f^{a} Y_{a}(e, \omega, f)+i_{\xi} h^{a} Z_{a}(e, \omega, f) .
$$

So, if we require that the Lagrangian be invariant under any arbitrary diffeomorphism for arbitrary $e^{a}, \omega^{a b}$, and $f^{a}$, then we must have

$$
\begin{aligned}
& D E_{a b}^{(\omega)}+e_{b} \wedge E_{a}^{(e)}+f_{b} \wedge E_{a}^{(f)}=0, \\
& X_{a}(\Phi)=0, \quad Y_{a}(\Phi)=0, \quad Z_{a}(\Phi)=0
\end{aligned}
$$

these equations are Bianchi identities. Considering that the Lagrangian is a Lorenz-diffeomorphism invariant quantity, then $\delta_{\xi} L=\mathfrak{L}_{\xi} L=\mathfrak{f}_{\xi} L=\mathrm{d} i_{\xi} L$. Therefore, Eq. (11) reduces to the following form:

$\mathrm{d} J=0$,

where $J$ is an off-shell current density $(n-1)$-form and it is expressed as follows:

$$
\begin{aligned}
J= & i_{\xi} e^{a} E_{a}^{(e)}+i_{\xi} f^{a} E_{a}^{(f)}+i_{\xi} h^{a} E_{a}^{(h)} \\
& +\left(i_{\xi} \omega^{a b}-\lambda^{a b}\right) E_{a b}^{(\omega)}+\Theta\left(\Phi, \delta_{\xi} \Phi\right)-i_{\xi} L .
\end{aligned}
$$

Since $J$ is a closed form, by virtue of [35], we can write $J$ as an exact form, that is, $J=d K$. By varying (15) thus we will find the following expression for the symplectic current:

$$
\begin{aligned}
\Omega\left(\Phi, \delta \Phi, \delta_{\xi} \Phi\right)= & \delta \Theta\left(\Phi, \delta_{\xi} \Phi\right)-\delta_{\xi} \Theta(\Phi, \delta \Phi) \\
= & \mathrm{d}\left(\delta K-i_{\xi} \Theta(\Phi, \delta \Phi)\right)-i_{\xi} e^{a} \delta E_{a}^{(e)} \\
& -i_{\xi} f^{a} \delta E_{a}^{(f)}-i_{\xi} h^{a} \delta E_{a}^{(h)} \\
& -\delta e^{a} \wedge i_{\xi} E_{a}^{(e)}-\delta f^{a} \wedge i_{\xi} E_{a}^{(f)}
\end{aligned}
$$

$$
\begin{aligned}
& -\delta h^{a} \wedge i_{\xi} E_{a}^{(h)}-\delta \omega^{a b} \wedge i_{\xi} E_{a b}^{(\omega)} \\
& -\left(i_{\xi} \omega^{a b}-\lambda^{a b}\right) \delta E_{a b}^{(\omega)}+\lambda^{a b} \delta E_{a b}^{(\omega)} .
\end{aligned}
$$

The symplectic current is linear with respect to $\delta_{\xi} \Phi^{a}$, and the $\delta_{\xi} \Phi^{a}$ become zero when $\xi$ is a Killing vector field. If we require that $\xi$ be a Killing vector field then $\Omega$ becomes zero. Therefore, in the first order formalism the ADT conserved current is defined as

$$
\begin{aligned}
J_{\mathrm{ADT}}= & i_{\xi} e^{a} \delta E_{a}^{(e)}+i_{\xi} f^{a} \delta E_{a}^{(f)}+i_{\xi} f^{a} \delta E_{a}^{(f)} \\
& +\left(i_{\xi} \omega^{a b}-\lambda^{a b}\right) \delta E_{a b}^{(\omega)}-\lambda^{a b} \delta E_{a b}^{(\omega)} \\
& +\delta e^{a} \wedge i_{\xi} E_{a}^{(e)}+\delta f^{a} \wedge i_{\xi} E_{a}^{(f)}+\delta h^{a} \wedge i_{\xi} E_{a}^{(h)} \\
& +\delta \omega^{a b} \wedge i_{\xi} E_{a b}^{(\omega)} \\
= & \mathrm{d}\left(\delta K-i_{\xi} \Theta(\Phi, \delta \Phi)\right)
\end{aligned}
$$

We know that the relation between the ADT conserved current and the ADT conserved charge is $J_{\mathrm{ADT}}=\mathrm{d} Q_{\mathrm{ADT}}$, so the ADT conserved charge is given by

$Q_{\mathrm{ADT}}=\delta K-i_{\xi} \Theta(e, \omega ; \delta e, \delta \omega)$.

Now, we consider an one-parameter path in the solution space. For this purpose, suppose that the $\Phi(\mathcal{Q})$ are a collection of fields which solve the equations of motion of the considered theory, where $\mathcal{Q}$ is a free parameter in the solution space of the equations of motion. Now, we replace $\mathcal{Q}$ by ${ }_{s} \mathcal{Q}$, where $0 \leq s \leq 1$ is a parameter. By expanding $\Phi(s \mathcal{Q})$ in terms of $s$ we have $\Phi(s \mathcal{Q})=\Phi(0)+\Phi^{\prime}(0) s+\cdots$, where the prime denotes differentiation with respect to $s$. Then, by substituting $\Phi=\Phi(s \mathcal{Q})$ and $\delta \Phi=\Phi^{\prime}(0)$ into Eq. (18), we can define the quasi-local conserved charge associated to the Killing vector field $\xi$ as $[8,9]$

$Q(\xi)=c \int_{0}^{1} \mathrm{~d} s \int_{\Sigma} Q_{\mathrm{ADT}}(e, \omega \mid s)$,

where $c$ is a normalization factor and $\Sigma$ is a time-like $(n-2)$ surface, also, $s=0$ and $s=1$ correspond to the background solution and the solution of interest, respectively. Therefore, integration over $s$ is just integration over a one-parameter path in the solution space. By substituting (18) into (19), we obtain the following expression for the conserved charge associated to the Killing vector field $\xi$ :

$Q(\xi)=c \int_{\Sigma}\left(\Delta K-i_{\xi} \int_{0}^{1} \mathrm{~d} s \Theta(e, \omega \mid s)\right)$,

where $\Delta K=K_{s=1}(\xi)-K_{s=0}(\xi)$. In this way, we find an expression for the conserved charge which is does not depend on the $(n-2)$-surface $\Sigma$. So, we can choose $\Sigma$ everywhere and then we obtain the conserved charges of spacetimes which are not asymptotically flat, nor even AdS.

As we have mentioned in introduction, our approach has some similarity with the framework of Poincare gauge theory [27-32] (see also [36-38], where the authors have studied the 
conserved charges in the framework of Poincaré gauge theory). The difference between this approach and the Poincaré gauge theory is that we combined the Lorenz gauge transformation with a diffeomorphism and introduced the total variation under a Lorenz-diffeomorphism transformation. In this way, we have a unique transformation and using it we can obtain the conserved charges of a covariant theory of gravity in the first order formalism. This method provides a derivation of the entropy formula for black hole solutions in gravity theories defined by a Chern-Simons gravitational action in $3 D$ [26]. It was pointed out in Ref. [23] that the derivation of the classical Wald formula for the entropy is problematic in the first order formalism using the spin connection. But by introduction the Lorentz-Lie derivative, according to our approach one can overcome this difficulty.

\section{Quasi-local conserved charges of the Lovelock gravity}

In this section, we apply the above procedure to the Lovelock theory in arbitrary dimensions and we will find a general expression for the conserved charges of this theory in any dimension. This theory was first proposed by Lovelock [39]; it has the same degrees of freedom as general relativity and it is ghost-free. The Lovelock Lagrangian is given by [40]

$L(e, R)=\sum_{p=0}^{[n / 2]} \alpha_{p} L^{(p)}$

where

$L^{(p)}=\varepsilon_{a_{1} \cdots a_{n}} R^{a_{1} a_{2}} \wedge \cdots \wedge R^{a_{2 p-1} a_{2 p}} \wedge e^{a_{2 p+1}} \wedge \cdots \wedge e^{a_{n}}$.

In the above Lagrangian, the $\alpha_{p}$ are arbitrary dimensionful coupling constants and $[x]$ denotes the integer part of $x$. Because the procedure which leads to Eq. (20) is linear in $L$, we consider $L^{(p)}$.

After some calculations, for the Lagrangian Eq. (21), we find

$E_{a_{n}}^{(e)}=\frac{\partial L^{(p)}}{\partial e^{a_{n}}}, \quad E_{a_{1} a_{2}}^{(\omega)}=D \frac{\partial L^{(p)}}{\partial R^{a_{1} a_{2}}}$,

$\Theta^{(p)}(e, \omega ; \delta \omega)=\delta \omega^{a_{1} a_{2}} \wedge \frac{\partial L^{(p)}}{\partial R^{a_{1} a_{2}}}$,

where

$$
\begin{aligned}
\frac{\partial L^{(p)}}{\partial e^{a_{n}}}= & (n-2 p) \varepsilon_{a_{1} \cdots a_{n}} e^{a_{n-1}} \wedge \cdots \wedge e^{a_{2 p+1}} \\
& \wedge R^{a_{1} a_{2}} \cdots \wedge R^{a_{2 p-1} a_{2 p}}, \\
\frac{\partial L^{(p)}}{\partial R^{a_{1} a_{2}}}= & p \varepsilon_{a_{1} a_{2} \cdots a_{n}} R^{a_{3} a_{4}} \wedge \cdots \wedge R^{a_{2 p-1} a_{2 p}} \\
& \wedge e^{a_{2 p+1}} \wedge \cdots \wedge e^{a_{n}} .
\end{aligned}
$$

By substituting Eqs. (22), (23), and (24) into Eq. (15), we can read off $K^{(p)}$ as follows:

$K^{(p)}=\left(i_{\xi} \omega^{a_{1} a_{2}}-\lambda^{a_{1} a_{2}}\right) \frac{\partial L^{(p)}}{\partial R^{a_{1} a_{2}}}$.

Now, we can calculate the contribution of the $p$-term in the conserved charge $Q^{(p)}(\xi)$ using Eq. (19) and then the conserved charge is $Q(\xi)=\sum_{p=0}^{[n / 2]} \alpha_{p} Q^{(p)}(\xi)$. Thus what we found here is an off-shell quasi-local conserved charge and it is exactly the ADT charge for any solution with any asymptotical behavior which admits the Killing vector field $\xi$. Also, as we mentioned and deduced earlier, we can calculate this charge on any time-like codimension- 2 surface.

\section{Application to the BHT gravity}

\subsection{BHT gravity and its conserved charges}

Bergshoeff-Hohm-Townsend (BHT) gravity is a covariant gravity theory in three dimensions [41]. In three dimensions, it is convenient to define a dualized spin connection and a dualized curvature 2-form as

$\omega^{a}=\frac{1}{2} \varepsilon_{b c}^{a} \omega^{b c}, \quad R^{a}=\frac{1}{2} \varepsilon_{b c}^{a} R^{b c}$,

respectively, where $\varepsilon_{a b c}$ is the Levi-Civita symbol in 3D. The Lagrangian of BHT gravity is given by (for instance, see [42])

$$
\begin{aligned}
L= & -\sigma e^{a} \wedge R_{a}+\frac{\Lambda_{0}}{6} \varepsilon_{a b c} e^{a} \wedge e^{b} \wedge e^{c} \\
& +\frac{1}{m^{2}}\left(f^{a} \wedge R_{a}+\varepsilon_{a b c} e^{a} \wedge f^{b} \wedge f^{c}\right)+h^{a} \wedge T_{a}
\end{aligned}
$$

where $\sigma, m$, and $\Lambda_{0}$ are the sign, the mass parameter, and the cosmological parameter, respectively. The equations of motion of BHT gravity are

$$
\begin{aligned}
E_{a}^{(e)}= & -\sigma R_{a}+\frac{\Lambda_{0}}{2} \varepsilon_{a b c} e^{b} \wedge e^{c}+D h_{a} \\
& -\frac{1}{2 m^{2}} \varepsilon_{a b c} f^{b} \wedge f^{c}=0 \\
E_{a}^{(\omega)}= & -\sigma T_{a}-\frac{1}{m^{2}} D f_{a}+\varepsilon_{a b c} e^{b} \wedge h^{c}=0 \\
E_{a}^{(f)}= & -\frac{1}{m^{2}}\left(R_{a}+\varepsilon_{a b c} e^{b} \wedge f^{c}\right)=0 \\
E_{a}^{h}= & T_{a}=0,
\end{aligned}
$$

where $D$ denotes the exterior covariant derivative, and the surface term of this theory is

$\Theta(\Phi, \delta \Phi)=-\sigma \delta \omega^{a} \wedge e_{a}-\frac{1}{m^{2}} \delta \omega^{a} \wedge f_{a}+\delta e^{a} \wedge h_{a}$ 
It is obvious that this theory is torsion-free. One can solve the equations of motion (29) and find

$h^{a}=-\frac{1}{m^{2}} C^{a}, \quad f^{a}=-S^{a}$.

In the above equations $S_{\mu \nu}=\mathcal{R}_{\mu \nu}-\frac{1}{4} g_{\mu \nu} \mathcal{R}$ is the $3 \mathrm{D}$ Schouten tensor and $C_{\mu \nu}=\sqrt{-g} \varepsilon_{\nu \alpha \beta} \nabla^{\alpha} S_{\mu}^{\beta}$ is the Cotton tensor, where $\mathcal{R}_{\mu \nu}$ and $\mathcal{R}$ are, respectively, the Ricci tensor and the Ricci scalar. One can calculate Eq. (15) to find $K(\xi)$ for the BHT gravity:

$K(\xi)=-\sigma\left(i_{\xi} \omega^{a}-\chi_{\xi}^{a}\right) e_{a}-\frac{1}{m^{2}}\left(i \xi \omega^{a}-\chi_{\xi}^{a}\right) f_{a}+i_{\xi} e^{a} h_{a}$,

where $\chi_{\xi}^{a}$ is the dual of the generator of the Lorenz gauge transformations $\lambda_{\xi}^{a b}$,

$\chi_{\xi}^{a}=\frac{1}{2} \varepsilon_{b c}^{a} \lambda_{\xi}^{b c}$.

By substituting Eqs. (30) and (33) into Eq. (18) we find

$$
\begin{aligned}
Q_{\mathrm{ADT}}(\xi)= & i_{\xi} e^{a} \delta h_{a}+i_{\xi} h^{a} \delta e_{a}-\sigma i_{\xi} e^{a} \delta \omega_{a} \\
& -\frac{1}{m^{2}} i_{\xi} f^{a} \delta \omega_{a}-\sigma\left(i_{\xi} \omega^{a}-\chi_{\xi}^{a}\right) \delta e_{a} \\
& -\frac{1}{m^{2}}\left(i_{\xi} \omega^{a}-\chi_{\xi}^{a}\right) \delta f_{a} .
\end{aligned}
$$

Since $\lambda^{a b}=e^{\sigma[a} £_{\xi} e_{\sigma}^{b]}$ so $\chi_{\xi}^{a}$ is given as [26]

$\chi_{\xi}^{a}=i_{\xi} \omega^{a}-\frac{1}{2} \varepsilon_{b c}^{a} e^{\nu b}\left(i_{\xi} T^{c}\right)_{\nu}+\frac{1}{2} \varepsilon_{b c}^{a} e_{\mu}^{b} e_{\nu}^{c} \nabla^{\mu} \xi^{\nu}$,

and because the BHT gravity is torsion-free, we have

$i_{\xi} \omega^{a}-\chi_{\xi}^{a}=-\frac{1}{2} \varepsilon_{b c}^{a} e_{\mu}^{b} e_{\nu}^{c} \nabla^{\mu} \xi^{\nu}$.

By substituting Eq. (36) into Eq. (19), one can find the conserved charge of a considered solution associated to a Killing vector $\xi$.

\subsection{Rotating OTT black hole solution}

The BHT gravity admits the $\mathrm{AdS}_{3}$ spacetime as a unique maximally symmetric background when

$\sigma=1, \quad m^{2}=\frac{1}{2 l^{2}}, \quad \Lambda_{0}=-\frac{1}{2 l^{2}}$,

where $l$ is the $\mathrm{AdS}_{3}$ spacetime radius. Equations (36) are known as the BHT conditions. The rotating Oliva-TempoTronsoco (OTT) black hole spacetime solves the equations of motion of BHT gravity when the BHT conditions are satisfied. The rotating OTT black hole is defined by the metric [43-45]

$$
\begin{aligned}
\mathrm{d} s^{2}= & -N(r)^{2} F(r)^{2} \mathrm{~d} t^{2}+F(r)^{-2} d r^{2} \\
& +r^{2}\left(\mathrm{~d} \phi+N^{\phi}(r) \mathrm{d} t\right)^{2}
\end{aligned}
$$

where

$$
\begin{aligned}
& F(r)=\frac{H(r)}{r} \sqrt{\frac{H(r)^{2}}{l^{2}}+\frac{b}{2} H(r)(1+\eta)+\frac{b^{2} l^{2}}{16}(1-\eta)^{2}-\mu \eta} \\
& N(r)=1+\frac{b l^{2}}{4 H(r)}(1-\eta), \\
& N^{\phi}(r)=\frac{l}{2 r^{2}} \sqrt{1-\eta^{2}}(\mu-b H(r)), \\
& H(r)=\sqrt{r^{2}-\frac{\mu l^{2}}{2}(1-\eta)-\frac{b^{2} l^{4}}{16}(1-\eta)^{2} .}
\end{aligned}
$$

The metric (38) depends on three free parameters, $\mu, b$, and $\eta$. This metric reduces to the static OTT black hole metric when $\eta=1$ and for $b=0$, it represents the rotating BTZ black hole [46].

We can choose a dreibein

$$
\begin{gathered}
e^{0}=N(r) F(r) \mathrm{d} t, \quad e^{1}=F(r)^{-1} d r \\
e^{2}=r\left(\mathrm{~d} \phi+N^{\phi}(r) \mathrm{d} t\right) .
\end{gathered}
$$

It should be noted here that the Cotton tensor vanishes for this solution.

Now, we take the $\mathrm{AdS}_{3}$ spacetime as a background solution using the following dreibeins:

$\bar{e}^{0}=\frac{r}{l} \mathrm{~d} t, \quad \bar{e}^{1}=\frac{l}{r} d r, \quad \bar{e}^{2}=r \mathrm{~d} \phi$,

i.e. the $\mathrm{AdS}_{3}$ spacetime corresponds to $s=0$. We take the integration surface $\Sigma$ as a circle with a radius at infinity. It can be shown that Eq. (34) on $\Sigma$ reduces to

$Q_{\mathrm{ADT}}(\xi)=\left\{-2 i_{\xi} \bar{e}_{a} \delta \omega_{\phi}^{a}+2 l^{2}\left(i_{\xi} \bar{\omega}^{a}-\bar{\chi}_{\xi}^{a}\right) \delta S_{a \phi}\right\} \mathrm{d} \phi$,

for the rotating OTT black hole solution.

The energy corresponds to the Killing vector $\xi_{t}=\partial_{t}$. For this Killing vector on the background, Eq. (36) becomes

$i_{\xi(t)} \bar{\omega}^{a}-\bar{\chi}_{\xi(t)}^{a}=\frac{1}{l^{2}} e_{\phi}^{a}$.

By substituting Eqs. (41) and (43) into Eq. (42), we have

$Q_{\mathrm{ADT}}(\xi)=2 r\left\{\frac{1}{l} \delta \omega_{\phi}^{0}+\delta S_{\phi}^{2}\right\} \mathrm{d} \phi$,

and by integrating over a one-parameter path on the solution space, we find

$$
\begin{aligned}
\int_{0}^{1} Q_{\mathrm{ADT}}(\xi) \mathrm{d} s= & 2 r\left\{\frac{1}{l}\left[\omega_{\phi(s=1)}^{0}-\omega_{\phi(s=0)}^{0}\right]\right. \\
& \left.+\left[S_{\phi(s=1)}^{2}-S_{\phi(s=0)}^{2}\right]\right\} \mathrm{d} \phi
\end{aligned}
$$


where $s=1$ corresponds to the rotating OTT black hole solution. By expanding $\omega_{\phi(s=1)}^{0}$ and $S_{\phi(s=1)}^{2}$ about infinity, we have

$$
\begin{aligned}
\omega_{\phi(s=1)}^{0}= & \omega_{\phi(s=0)}^{0}+\frac{b l}{4}(1+\eta)-\frac{l}{16 r}\left[b^{2} l^{2}\left(1+\eta^{2}\right)+8 \mu\right] \\
& +\mathcal{O}\left(r^{-2}\right), \\
S_{\phi(s=1)}^{2}= & S_{\phi(s=0)}^{2}-\frac{b}{4}(1+\eta)-\frac{b^{2} l^{2}}{16 r}\left(1-\eta^{2}\right) \\
& +\mathcal{O}\left(r^{-2}\right)
\end{aligned}
$$

therefore Eq. (45) becomes

$$
\int_{0}^{1} Q_{\mathrm{ADT}}\left(\partial_{t}\right) \mathrm{d} s=-\left\{\mu+\frac{1}{4} b^{2} l^{2}+\mathcal{O}\left(r^{-1}\right)\right\} \mathrm{d} \phi .
$$

By substituting Eq. (47) into Eq. (19) and by taking $r \rightarrow \infty$, we find the energy of the rotating OTT black hole,

$E=\frac{1}{4}\left(\mu+\frac{1}{4} b^{2} l^{2}\right)$.

In this subsection, we choose the normalization factor in (19) as $c=-\frac{1}{8 \pi}$. Now, we take $\xi_{(\phi)}=\partial_{\phi}$ and it is straightforward to show that

$i_{\xi_{(\phi)}} \bar{\omega}^{a}-\bar{\chi}_{\xi_{(\phi)}}^{a}=e_{t}^{a}$.

In this case, Eq. (42) reduces to

$Q_{\mathrm{ADT}}(\xi)=-2 r\left\{\delta \omega_{\phi}^{2}+l \delta S_{\phi}^{0}\right\} \mathrm{d} \phi$.

By expanding $\omega_{\phi(s=1)}^{2}$ and $S_{\phi(s=1)}^{0}$ about infinity, we obtain

$$
\begin{aligned}
\omega_{\phi(s=1)}^{2}= & \omega_{\phi(s=0)}^{2}-\frac{b l}{4} \sqrt{1-\eta^{2}}+\frac{l}{16 r}\left[b^{2} l^{2}(1-\eta)+8 \mu\right] \\
& \times \sqrt{1-\eta^{2}}+\mathcal{O}\left(r^{-2}\right) \\
S_{\phi(s=1)}^{0}= & S_{\phi(s=0)}^{0}+\frac{b}{4} \sqrt{1-\eta^{2}} \\
& +\frac{b^{2} l^{2}}{16 r}(1+\eta) \sqrt{1-\eta^{2}}+\mathcal{O}\left(r^{-2}\right) .
\end{aligned}
$$

In a similar way to the energy, we can find the angular momentum of the rotating OTT black hole as

$j=l \sqrt{1-\eta^{2}} E$.

Now, we want to find the entropy of the rotating OTT black hole. The BHT gravity is a Chern-Simons-like theory of gravity. In Ref. [26], we have found a general formula for the Chern-Simons-like theories of gravity. Using the formula for the entropy of black hole solutions in a generalized massive gravity theory obtained in [26], and noticing that generalized massive gravity reduces to BHT gravity when the mass parameter of the Lorenz Chern-Simons term tends to infinity, one can calculate the entropy of the black hole solutions of the BHT gravity by the following formula:

$S=\frac{1}{4} \int_{0\left(r=r_{h}\right)}^{2 \pi} \frac{\mathrm{d} \phi}{\sqrt{g_{\phi \phi}}}\left(g_{\phi \phi}-\frac{1}{m^{2}} S_{\phi \phi}\right)$,

where $r_{h}$ is the radius of the Killing horizon. The Killing horizon of the OTT black hole is located at $r_{h}=r_{+}$and $r_{+}$ is given by [47]

$r_{+}=l \sqrt{\frac{1+\eta}{2}}\left(-\frac{b l}{2} \sqrt{\eta}+\sqrt{\mu+\frac{1}{4} b^{2} l^{2}}\right)$.

By some calculations, one can show that

$$
\begin{aligned}
S_{\phi \phi}= & -\frac{1}{2}\left(\frac{H^{2}}{l^{2}}+\frac{b}{2} H(1+\eta)+\frac{\mu}{2}(1-\eta)\right. \\
& \left.-\frac{b^{2} l^{2}}{16}(1+\eta)^{2}+\frac{b^{2} l^{2}}{4}\right) .
\end{aligned}
$$

By substituting the above component of the Schouten tensor into Eq. (53), the entropy of an OTT black hole can be obtained:

$S=2 \pi l \sqrt{\frac{(1+\eta) E}{2}}$.

Fortunately our obtained results (48), (52), and (56) for the energy, angular momentum, and entropy, respectively, exactly match with the results of Ref. [47]. So these calculations indicate that our method for calculations of conserved charges for these types of black hole solutions works as well.

\section{Conclusion}

There are several approaches to obtain the mass and angular momentum of black holes for higher curvature theories [1-18]. The authors of [8] have obtained the quasi-local conserved charges for black holes in any diffeomorphically invariant theory of gravity. By considering an appropriate variation of the metric, they have established a one-to-one correspondence between the ADT approach and the linear Noether expressions.

In this paper we have found quasi-local conserved charges of covariant theories of gravity in the first order formalism. In the first order formalism of gravity, one can use the concept of the combined Lorenz-diffeomorphism symmetry to obtain the conserved charges of a gravity theory. Using this, we have found a general formula (20) for the quasi-local conserved charges in the first order formalism. In this way, we can calculate the conserved charges of any solution of a gravity theory which is not an asymptotically AdS or flat spacetime. Then we have simplified the resulting formula for the Lovelock theory of gravity. Using the provided formalism we found the energy and angular momentum of the rotating 
OTT black hole solution of BHT gravity. Then we calculated the entropy of the rotating OTT black hole using the general formula for the Chern-Simons-like theories of gravity [26]. Our results for the energy, the angular momentum, and the entropy exactly coincide on the results for these quantities which were presented in Ref. [47].

Acknowledgments M. R. Setare thanks Y. Obukhov and T. Jacobson for helpful comments and discussions.

Open Access This article is distributed under the terms of the Creative Commons Attribution 4.0 International License (http://creativecomm ons.org/licenses/by/4.0/), which permits unrestricted use, distribution, and reproduction in any medium, provided you give appropriate credit to the original author(s) and the source, provide a link to the Creative Commons license, and indicate if changes were made. Funded by SCOAP ${ }^{3}$.

\section{References}

1. L.F. Abbott, S. Deser, Nucl. Phys. B 195, 76 (1982)

2. L.F. Abbott, S. Deser, Phys. Lett. B 116, 259 (1982)

3. R.L. Arnowitt, S. Deser, C.W. Misner, Gen. Rel. Grav. 40, 1997 (2008)

4. S. Deser, B. Tekin, Phys. Rev. D 67, 084009 (2003)

5. S. Deser, B. Tekin, Phys. Rev. Lett. 89, 101101 (2002)

6. C. Senturk, T.C. Sisman, B. Tekin, Phys. Rev. D 86, 124030 (2012)

7. J.D. Brown, J.W. York, Phys. Rev. D 47, 1407 (1993)

8. W. Kim, S. Kulkarni, S.H. Yi, Phys. Rev. Lett. 111, 081101 (2013)

9. W. Kim, S. Kulkarni, S.H. Yi, Phys. Rev. D 88, 124004 (2013)

10. A. Bouchareb, G. Clement, Class. Quant. Grav. 24, 5581 (2007)

11. S. Hyun, J. Jeong, S.A. Park, S.H. Yi, Phys. Rev. D 90, 104016 (2014)

12. G. Clement, Phys. Rev. D 49, 5131 (1994)

13. G. Clement, Class. Quant. Grav. 11, L115 (1994)

14. O. Miskovic, R. Olea, JHEP 0912, 046 (2009)

15. G. Giribet, M. Leston, JHEP 1009, 070 (2010)

16. O. Hohm, E. Tonni, JHEP 1004, 093 (2010)

17. S. Nam, J.D. Park, S.H. Yi, JHEP 1007, 058 (2010)
18. S. Nam, J.D. Park, S.H. Yi, Phys. Rev. D 82, 124049 (2010)

19. M.R. Setare, H. Adami, Phys. Lett. B 774, 280 (2015)

20. R.M. Wald, A. Zoupas, Phys. Rev. D 61, 084027 (2000)

21. R.M. Wald, Phys. Rev. D 48, 3427 (1993)

22. Y. Tachikawa, Class. Quantum Gravity 24, 737 (2007)

23. T. Jacobson, A. Mohd, Phys. Rev. D 92, 124010 (2015)

24. Y.N. Obukhov, G.F. Rubilar, Phys. Rev. D 74, 064002 (2006)

25. K. Yano, The Theory of Lie Derivatives and its Applications (NorthHolland, Amsterdam, 1955)

26. M.R. Setare, H. Adami, Nucl. Phys. B 902, 115 (2016)

27. T.W.B. Kibble, J. Math. Phys. 2, 212 (1961)

28. D. W. Sciama, On the analogy between charge and spin in general relativity, in: Recent Developments in General Relativity, Festschrift for Infeld (Pergamon Press, Oxford; PWN, Warsaw, 1962) pp. 415-439

29. Milutin Blagojević, Gravitation and Gauge Symmetries IOP Publishing, Bristol (2002)

30. Y.N. Obukhov, Int. J. Geom. Meth. Mod. Phys. 3, 95 (2006)

31. Y.N. Obukhov, G.F. Rubilar, Phys. Rev. D 76, 124030 (2007)

32. M. Blagojević, F.W. Hehl (eds.), Gauge Theories of Gravitation, A Reader with Commentaries (Imperial College Press, London, 2013)

33. M. Blagojević, B. Cvetković, arXiv:1310.8309 [gr-qc]

34. M. Blagojević, B. Cvetković, Phys. Rev. D 92, 024047 (2015)

35. R. M. Wald, J. Math. Phys. 31, 2378 (1990)

36. P. Baecler, R. Hecht, F.W. Hehl, T. Shirafuji, Prog. Theor. Phys. 78, 16 (1987)

37. T. Kawai, Prog. Theor. Phys. 79, 920 (1988)

38. C.M. Chen, J.M. Nester, R.S. Tung, Int. J. Mod. Phys. D 24, $1530026(2015)$

39. D. Lovelock, J. Math. Phys. 12, 498 (1971)

40. F. Correa, M. Hassaine, JHEP 1402, 014 (2014)

41. E.A. Bergshoeff, O. Hohm, P.K. Townsend, Phys. Rev. Lett. 102, 201301 (2009)

42. M. Blagojević, B. Cvetković, JHEP 1101, 082 (2011)

43. J. Oliva, D. Tempo, R. Tronsoco, JHEP 07, 011 (2009)

44. G. Giribet, J. Oliva, D. Tempo, R. Troncoso, Phys. Rev. D 80, 124046 (2009)

45. G. Giribet, M. Leston, JHEP 09, 070 (2010)

46. M. Banados, C. Teitelboim, J. Zanelli, Phys. Rev. Lett. 69, 1849 (1992)

47. M. Blagojević, B. Cvetković, Phys. Rev. D 93, 044018 (2016) 